\title{
Astragalus polysaccharides combined with ibuprofen exhibit a therapeutic effect on septic rats via an anti-inflammatory cholinergic pathway
}

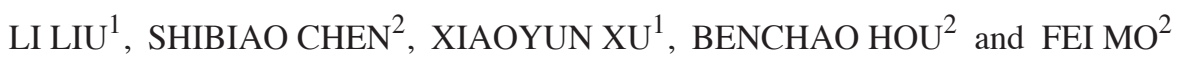 \\ ${ }^{1}$ School of Medicine, Graduate School, Nanchang University; ${ }^{2}$ Department of Anesthesia, \\ The First Affiliated Hospital, Nanchang University, Nanchang, Jiangxi 330006, P.R. China
}

Received March 14, 2016; Accepted March 06, 2017

DOI: $10.3892 /$ etm.2017.4865

\begin{abstract}
The aim of the present study was to investigate the effects of Astragalus polysaccharides (APS) in combination with ibuprofen (IBU) in the treatment of sepsis and the underlying mechanism. The combined drug treatment was evaluated in a rat model of cecal ligation and puncture (CLP). The serum concentrations of tumor necrosis factor (TNF)- $\alpha$, interleukin (IL)-6 and acetylcholine (ACh) were determined. Nicotinic acetylcholine (nAChR) $\alpha 7$ receptor expression and histopathological changes in the lung tissue were also observed. When compared with untreated rats and rats treated with either component alone, the combined treatment significantly decreased the production of TNF- $\alpha$ and IL-6 $(\mathrm{P}<0.05)$, and increased $\mathrm{nAChR} \alpha 7$ receptor mRNA expression and the release of $\mathrm{ACh}$ in the serum $(\mathrm{P}<0.05)$. These results demonstrated that APS combined with IBU can effectively reduce the generation of inflammatory mediators in the serum of CLP-induced septic rats. These effects may be mediated via a cholinergic anti-inflammatory pathway involving $\mathrm{AChR} \alpha 7$.
\end{abstract}

\section{Introduction}

Sepsis is serious condition caused by an infection combined with systemic inflammatory response syndrome and has a mortality rate as high as $30-50 \%$, which is associated with lack of hemodynamic stability, abnormal platelets, multiple organ dysfunction and disseminated intravascular coagulation $(1,2)$. These pathophysiological processes involve the uncontrolled overexpression of inflammatory cytokines, including tumor necrosis factor (TNF)- $\alpha$ and interleukin (IL)-6.

Correspondence to: Dr Fei Mo, Department of Anesthesia, The First Affiliated Hospital, Nanchang University, 17 Yongwai Zheng Street, Nanchang, Jiangxi 330006, P.R. China

E-mail: mofei11111@hotmail.com

Key words: ibuprofen, Astragalus polysaccharides, cecal ligation and puncture, cholinergic anti-inflammatory pathway
Chinese medicines have been demonstrated to be effective systemic treatments for sepsis $(3,4)$. Astragalus polysaccharide (APS) is the main biologically active ingredient of Astragalus, a valuable traditional Chinese medicine that boosts immune function and suppresses inflammation $(5,6)$. Previous studies suggest that the anti-inflammatory and immune-regulating function of certain Chinese medicines is associated with their ability to inhibit inflammatory cytokine synthesis (3-6). Ibuprofen (IBU), a cyclooxygenase inhibitor with anti-inflammatory and anti-rheumatic activity, has been used for the treatment of respiratory tract infections and acute lung injury with few side effects (7). Previous studies have demonstrated that the immune activator APS in combination with the cyclooxygenase inhibitor IBU not only increases macrophage phagocytosis, but also significantly inhibits the secretion of prostaglandin E2 and IL-1 $(8,9)$.

The cholinergic anti-inflammatory pathway (CAP) is important in inflammatory processes, and has major implications for immunology and therapeutics (10). The nicotinic acetylcholine receptor (nAChR) $\alpha 7$, a member of a family of ligand-gated, pentameric ion channels, is essential for the inhibition of cytokine synthesis by the CAP (11).

The present study aimed to investigate the effect of APS combined with IBU on TNF- $\alpha$ and IL- 6 production in the serum of rats with cecal ligation and puncture (CLP)-induced sepsis. Furthermore, it aimed to demonstrate that this combination could be developed as a new treatment for sepsis. Since CAP has a critical role in regulating the generation of inflammatory cytokines during an inflammatory reaction, the effects of this drug combination on CAP-related signaling molecules were also examined in septic rats. It was hypothesized that the anti-inflammatory effect of APS combined with IBU may proceed via the CAP.

\section{Materials and methods}

Animals. A total of 40 specific-pathogen-free male SpragueDawley (SD) rats (weight, 250-300 g; age, 8-10 weeks) were obtained from the Department of Laboratory Animal Science of Nanchang University (Jiangxi, China). The production license number was 96021. All animal procedures were approved and conducted in accordance with the Guidelines for the Care 
and Use of Animals of the Ethics Committee of Nanchang University (Nanchang, China). Male Sprague-Dawley rats were housed in individual cages in a temperature-controlled room $\left(18-26^{\circ} \mathrm{C}, 40-60 \%\right.$ humidity) under a 12 -h light/dark cycle and with ad libitum access to food and water. The rats were sacrificed by tail vein air embolism following anesthetization with $1 \%$ pentobarbital sodium $(45 \mathrm{mg} / \mathrm{kg}$; Sinopharm Chemical reagent Co., Ltd., Shanghai, China).

General reagents and equipment. All reagents and assay kits were purchased from commercial sources. APS and IBU were purchased from Shanghai Yuanye Biotechnology Co., Ltd. (Shanghai, China) and Sigma-Aldrich, Inc. (Merck KGaA, Darmstadt, Germany), respectively. Rat IL-6 ELISA and TNF- $\alpha$ ELISA kits were purchased from Elabscience Biotechnology Co., Ltd. (Wuhan, China). Acetylcholine (ACh) assay kit was purchased from Nanjing Jiancheng Bioengineering Research Institute (Nanjing, China). Furthermore, the iMark microplate absorbance reader (Bio-Rad Laboratories, Inc., Hercules, CA, USA) and DNM-9602 microplate reader (Perlong Medical Equipment Co., Ltd., Nanjing, China) were used for microplate spectroscopic methods and ELISA. Finally, an optical microscope (BX45; Olympus Soft Imaging Solutions GmbH, Münster, Germany) was used for the observation of tissue sections.

Preparation of the rat model of CLP. Rats were anesthetized by an intraperitoneal injection of $45 \mathrm{mg} / \mathrm{kg}$ pentobarbital sodium. Polymicrobial sepsis was induced by CLP as described previously (12). The whole process was performed under sterile conditions with the abdominal skin sterilized by $75 \%$ alcohol. In brief, laparotomy was conducted through a $1.5-\mathrm{cm}$ lower-midline incision. The cecum was exposed and ligated immediately distal to the ileocecal valve to avoid intestinal obstruction, and was then punctured twice with a 16-gauge needle, squeezed gently to force out a small volume of feces and returned to the abdominal cavity. Finally, the abdomen was sutured layer-by-layer.

Grouping and surgical procedures. A total of $40 \mathrm{SD}$ rats were randomly divided into four groups: CLP (n=10), IBU $20 \mathrm{mg} / \mathrm{kg}$ $(\mathrm{n}=10)$, APS $25 \mathrm{mg} / \mathrm{kg}(\mathrm{n}=10)$ and IBU+APS $(\mathrm{n}=10)$ groups. APS was injected through the abdomen for 4 consecutive days prior to CLP. IBU was administered by injection at 2 and $8 \mathrm{~h}$ after CLP.

Blood sample and lung tissue collection. Blood samples of the rats in each group were collected from the femoral vein prior to and at 2,8 and $12 \mathrm{~h}$ after CLP. The samples were immediately treated with sodium citrate, and then centrifuged at $3,400 \times \mathrm{g}$ and $4^{\circ} \mathrm{C}$ (TGL-16; Changsha Xiangyi Centrifuge Instrument Co., Ltd., Changsha, China) for the separation of serum and plasma. The latter was stored at $-80^{\circ} \mathrm{C}$ for the measurement of ACh, TNF- $\alpha$ and IL- 6 . The left lungs were removed and rinsed several times in ice-cold saline, then dried with filter paper and stored at $-80^{\circ} \mathrm{C}$ for subsequent mRNA detection. The right lungs were cut into blocks and fixed with $10 \%$ neutral formalin. Pathological changes in the upper lobe were observed using a microscope after hematoxylin and eosin staining.
Detection of ACh, TNF- $a$ and IL-6. The ACh content of the samples was measured by a microplate spectroscopic method according to the manufacturer's protocol after rewarming the samples. Furthermore, TNF- $\alpha$ and IL- 6 were measured using ELISA kits, according to the manufacturer's instructions.

Reverse transcription-quantitative polymerase chain reaction (RT- $q P C R)$ assay of $\alpha 7$ receptor mRNA in lung tissues. The mRNA expression of the $\alpha 7$ receptor in lung tissues was analyzed by RT-qPCR. The total RNA was isolated from the lung tissues of each group using TRIzol reagent (Beyotime Institute of Biotechnology, Haimen, China) according to the manufacturer's instructions. In total, $5 \mu \mathrm{g}$ total RNA was reverse transcribed into cDNA using the PrimeScript ${ }^{\circledR} 1$ st Strand cDNA Synthesis kit (Takara Biotechnology Co., Ltd., Dalian, China) according to the manufacturer's protocol, and the PCR reaction mixtures were prepared using SYBR-Green qPCR master mix (Thermo Fisher Scientific, Inc., Waltham, MA, USA). $\beta$-actin was used as an internal control. The primers were synthesized by Sangon Biotech Co., Ltd., (Shanghai, China). The sequences were as follows: $\beta$-actin forward: 5'-AACCCTAAGGCCAACAGTGAAAAG-3' and reverse: 5'-TCATGAGGTAGTCTGTCAGGT-3'; $\alpha 7$ forward: 5'-AAACTCACAGATGGGCAAGG-3' and reverse: 5'-CCG TAAGCAACACGACTGAC-3'. The cycling conditions were as follows: 1 cycle at $95^{\circ} \mathrm{C}$ for $10 \mathrm{~min}$, followed by 40 cycles at $95^{\circ} \mathrm{C}$ for $15 \mathrm{sec}, 55^{\circ} \mathrm{C}$ for $20 \mathrm{sec}$ and $72^{\circ} \mathrm{C}$ for $1 \mathrm{~min}$. The relative expression level of $\alpha 7$ receptor mRNA in the lung tissues were determined by the $2^{-\Delta \Delta C q}$ method (13). The experiments were performed in triplicate.

Statistical analysis. SPSS 12.0 (SPSS, Inc., Chicago, IL, USA) was used for data analysis. All data are presented as the mean \pm standard deviation and were compared using Dunnett's multiple comparison tests. $\mathrm{P}<0.05$ was considered to indicate a statistically significant difference. The graphs and figures were created using Graphpad Prism 5 (GraphPad Software, Inc., La Jolla, CA, USA).

\section{Results}

APS combined with IBU reduces the generation of TNF- $\alpha$ and IL-6 in the serum of septic rats. As indicated in Figs. 1 and 2 , the TNF- $\alpha$ and IL-6 concentrations in the serum exhibited the same trend in each group after modeling: TNF- $\alpha$ and IL- 6 concentrations started to increase $2 \mathrm{~h}$ after CLP and peaked at $8 \mathrm{~h}$ after which their concentration declined slightly. TNF- $\alpha$ and IL- 6 in the serum of the APS, IBU and APS + IBU groups were reduced compared with those of the CLP group. However, only APS combined with IBU inhibited the increase of TNF- $\alpha$ and IL- 6 in the serum of CLP-induced septic rats significantly ( $\mathrm{P}<0.05$ vs. the CLP group). These results indicate that APS combined with IBU inhibits the generation of the inflammatory mediators TNF- $\alpha$ and IL- 6 during sepsis more strongly than either component alone and display superior anti-inflammatory effects.

APS combined with IBU increases the generation of ACh in the serum of septic rats. As shown in Fig. 3, the ACh levels in the serum differed at various time points after modeling. 


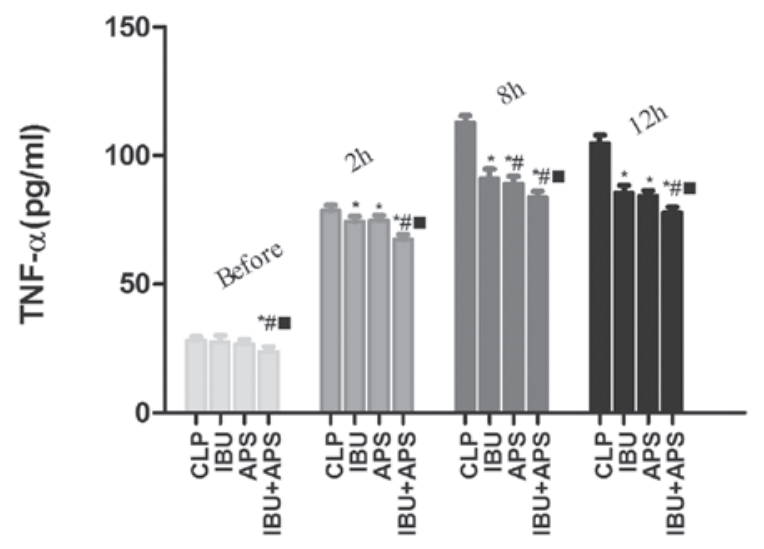

Figure 1. TNF- $\alpha$ expression detected by ELISA in the serum of septic rats at different timepoints (before and 2,8 and $12 \mathrm{~h}$ after CLP). Data are presented as the mean \pm standard deviation. ${ }^{*} \mathrm{P}<0.05$ vs. the CLP group; ${ }^{*} \mathrm{P}<0.05$ vs. the IBU group; " $\mathrm{P}<0.05$ vs. the APS group. TNF, tumor necrosis factor; CLP, cecal ligation and puncture; IBU, ibuprofen; APS, Astragalus polysaccharide.

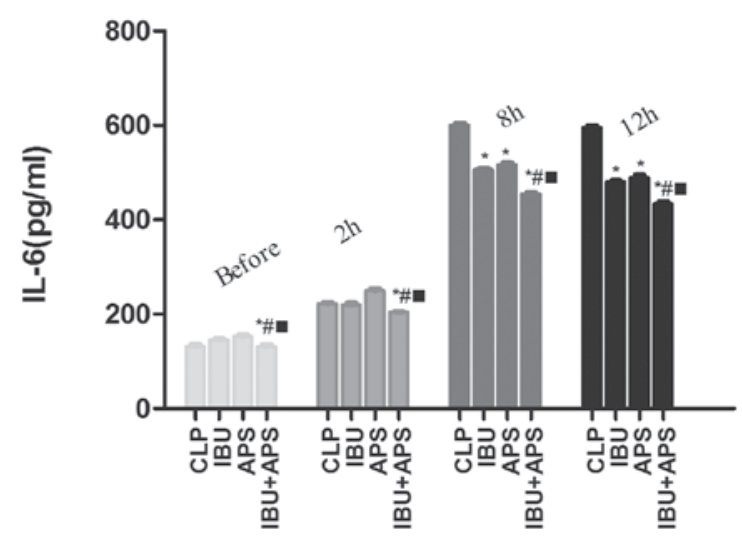

Figure 2. IL- 6 expression detected by ELISA in the serum of septic rats at different timepoints (before and 2, 8 and $12 \mathrm{~h}$ after CLP). Data are presented as the mean \pm standard deviation. ${ }^{*} \mathrm{P}<0.05$ vs. the CLP group; ${ }^{*} \mathrm{P}<0.05$ vs. the IBU group; "P $<0.05$ vs. the APS group. IL, interleukin; CLP, cecal ligation and puncture; IBU, ibuprofen; APS, Astragalus polysaccharide.

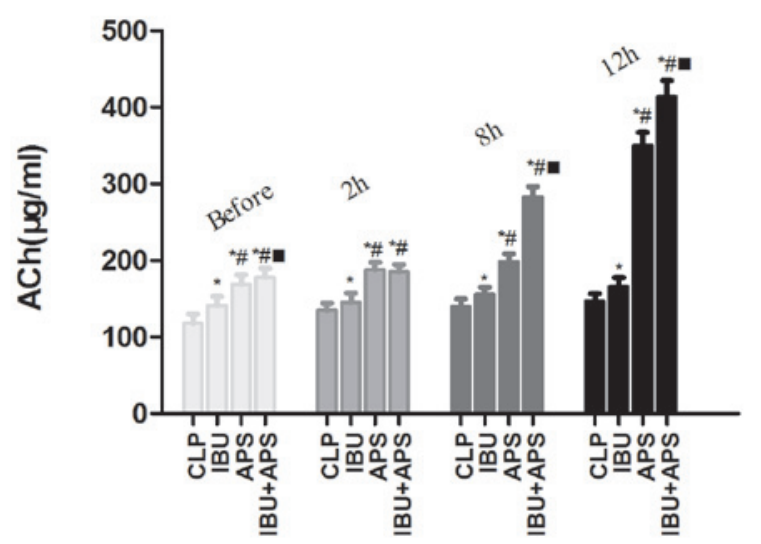

Figure 3. ACh concentration in the serum of septic rats at different timepoints (before and 2,8 and $12 \mathrm{~h}$ after CLP). Data are presented as the mean \pm standard deviation. ${ }^{*} \mathrm{P}<0.05$ vs. the CLP group; ${ }^{\#} \mathrm{P}<0.05$ vs. the IBU group; " $\mathrm{P}<0.05$ vs. the APS group. ACh, acetylcholine; CLP, cecal ligation and puncture; IBU, ibuprofen; APS, Astragalus polysaccharide.

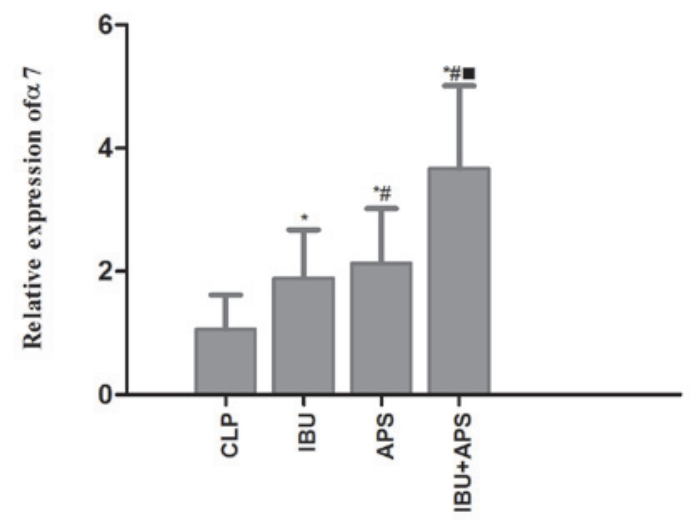

Figure 4. Expression of $\alpha 7$ receptor mRNA in the lung tissues of septic rats, measured by reverse transcription-quantitative polymerase chain reaction. Data are presented as the mean \pm standard deviation. ${ }^{*} \mathrm{P}<0.05$ vs. the CLP group; ${ }^{\#} \mathrm{P}<0.05$ vs. the IBU group; $" \mathrm{P}<0.05$ vs. the APS group. CLP, cecal ligation and puncture; IBU, ibuprofen; APS, Astragalus polysaccharide.
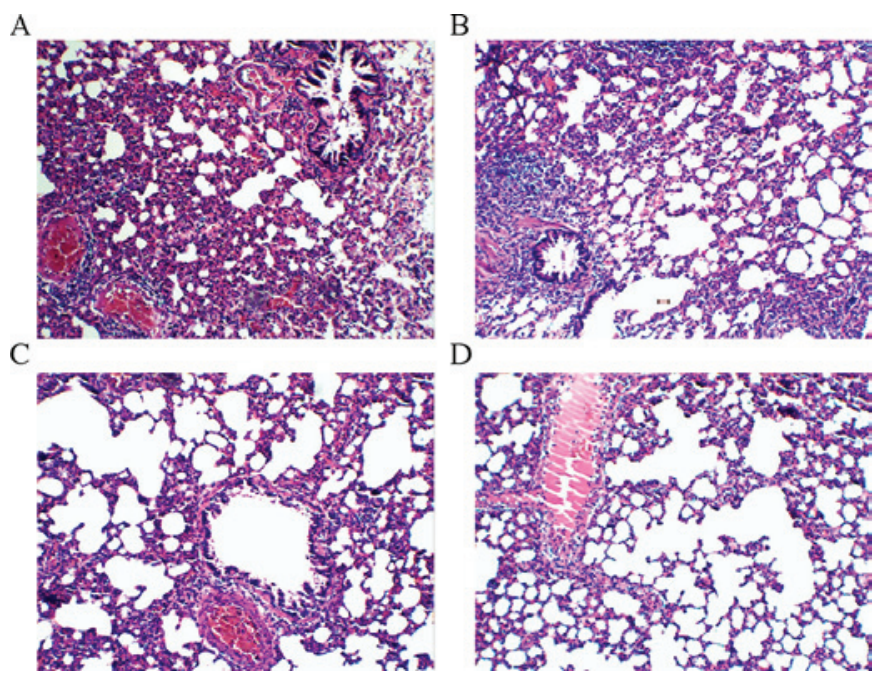

Figure 5. Pathological observations of the lung tissues from septic rats (A) Cecal ligation and puncture group, (B) ibuprofen group, (C) Astragalus polysaccharide group and (D) the combined therapy group. Hematoxylin and eosin staining (original magnification, x200).

Compared with the ACh levels in the serum of the CLP and the IBU groups, those in the APS + IBU groups were significantly increased $(\mathrm{P}<0.05)$. Furthermore, the increase was more evident in the combined therapies group. This suggests that the anti-septic effects of APS combined with IBU may be mediated by the CAP to some extent.

APS combined with IBU promotes the expression of $n A C h R$ $\alpha 7$ in the lung tissues of CLP-induced septic rats. Since $\mathrm{nAChR} \alpha 7$ is required for inhibiting cytokine synthesis by the CAP, the expression of $\mathrm{nAChR} \alpha 7$ was measured in the lung tissues of rats from the four groups. Compared with the CLP group, the IBU, APS and APS + IBU groups exhibited increased $\mathrm{nAChR} \alpha 7 \mathrm{mRNA}$ expression, and the expression level was significantly increased in the APS + IBU group $(\mathrm{P}<0.05$; Fig. 4). These results indicate that the CAP may be associated with the anti-inflammatory effect of APS combined with IBU. 
Pathological observations. Hematoxylin and eosin stained lung tissues in the CLP group showed collapsed alveoli, evident exudation, enlargement of the alveolar septum, and infiltration with numerous interstitial lung inflammatory cells when observed under a microscope (Fig. 5A). However, in the IBU and APS groups, the lung cells exhibited alveolar collapse, exudation, widening of the alveolar septum and some interstitial lung inflammatory cell infiltration (Fig. 5B and C). However, in the IBU + APS group the pathological features were clearly ameliorated (Fig. 5D), indicating a lower degree of pathological damage and a significantly improved lung histology.

\section{Discussion}

The present study provided novel evidence that APS in combination with IBU had more evident anti-inflammatory effects than either component used separately. APS combined with IBU effectively decreased the concentrations of IL- 6 and TNF- $\alpha$ in the serum of CLP-induced septic rats. Additionally, the combined drug treatment visibly improved the lung histology and reduced the degree of pathology of the CLP-induced septic rats. Furthermore, APS combined IBU also increased the expression of the $\alpha 7$ receptor and generation of ACh in the lung tissues of CLP-induced septic rats, indicating that the anti-inflammatory effect of in sepsis was dependent on the CAP.

Antibiotics are the most important treatment for sepsis, however over-prescribing leads to the emergence of more and more bacterial resistance. Therefore, clarifying the pathogenesis of sepsis and developing a safer and effective drug for its treatment has become a key challenge worldwide. Traditional Chinese medicines with anti-inflammatory effects serve as a potential source for the development of new treatments. For example, Xue et al (14) found that Chai Qin Cheng Qi Decoction relieved systemic inflammatory response syndrome and reduced the functional damage of organs in rats with severe acute pancreatitis.

Numerous studies have demonstrated that inflammatory cytokines, including TNF- $\alpha$ and IL- 6 , are important in the pathogenesis of sepsis and that an excessive inflammatory response can lead to acute lung injury (15). The extent of TNF- $\alpha$ and IL-6 expression is closely associated with the severity of sepsis $(16,17)$. The results of the present study showed that TNF- $\alpha$ and IL-6 started to increase $2 \mathrm{~h}$ after CLP and peaked at $8 \mathrm{~h}$, which is consistent with a previously published study. APS combined with IBU inhibited the increase of TNF- $\alpha$ and IL- 6 in the serum of CLP-induced septic rats, indicating that APS combined with IBU displayed detectable anti-inflammatory effects.

Studies on the molecular basis of the CAP have indicated that the $\mathrm{nAChR} \alpha 7$ subunit is important in mediating anti-inflammatory cytokine-suppressing effects $(10,11)$. Since the $\alpha 7$ receptor is required for ACh to inhibit TNF release (11), the effect of APS combined with IBU on this signaling molecule in CLP-induced septic rats was investigated in the present study. The combined drug treatment was demonstrated to increase ACh levels and the expression of the $\alpha 7$ receptor.

In summary, the present study demonstrated that APS combined with IBU can not only significantly reduce the generation of TNF- $\alpha$ and IL- 6 , but also increase the expression of the $\alpha 7$ receptor in the mRNA level and the release of
$\mathrm{ACh}$ in the serum of CLP-induced septic rats. These results indicate that the anti-inflammatory effects of APS and IBU may be medicated via the CAP and the $\alpha 7$ receptor. Therefore, APS in combination with IBU could be developed as a novel therapeutic regimen in the clinical therapy of sepsis.

\section{References}

1. Angus DC and Wax RS: Epidemiology of sepsis: An update. Crit Care Med 29 (7 Suppl): S109-S116, 2001.

2. Gu J, Sun P, Zhao H, Watts HR, Sanders RD, Terrando N, Xia P, Maze M and Ma D: Dexmedetomidine provides renoprotection against ischemia-reperfusion injury in mice. Crit Care 15: R153, 2011.

3. Liu H, Wu XZ and Li DH: Mechanism of Qing Re Jie Du Formula in Septic Rat Model. Chinese Journal of Surgery of Integrated Traditional and Western Medicine 6: 580-584, 2008 (In Chinese).

4. Li R, Chen WC, Wang WP, Tian WY and Zhang XG: Extraction, characterization of Astragalus polysaccharides and its immune modulating activities in rats with gastric cancer. Carbohydr Polym 78: 738-742, 2009.

5. Huang CL and Lu YP: Effect of Astragalus injection on insulin resistance in auxiliary treating patients with diabetes mellitus type 2. Zhongguo Zhong Xi Yi Jie He Za Zhi 23: 779-780, 2003 (In Chinese).

6. Tong Y and Hou H: Effects of Huangqi Guizhi Wuwu Tang on diabetic peripheral neuropathy. J Altern Complement Med 12: 506-509, 2006.

7. Bernard GR, Wheeler AP, Russell JA, Schein R, Summer WR, Steinberg KP, Fulkerson WJ, Wright PE, Christman BW, Dupont WD, et al: The effects of ibuprofen on the physiology and survival of patients with sepsis. The Ibuprofen in Sepsis Study Group. N Engl J Med 336: 912-918, 1997.

8. Chen SH WZ and Zhu PF: Joint research Astragalus polysaccharides and ibuprofen wound infections. Chinese Journal of Trauma 11: 356-358, 1995.

9. Lee KY and Jeon YJ: Macrophage activation by polysaccharide isolated from Astragalus membranaceus. Int Immunopharmacol 5: 1225-1233, 2005.

10. Pavlov VA and Tracey KJ: The cholinergic anti-inflammatory pathway. Brain Behav Immun 19: 493-499, 2005.

11. Wang H, Yu M, Ochani M, Amella CA, Tanovic M, Susarla S, Li JH, Wang H, Yang H, Ulloa L, et al: Nicotinic acetylcholine receptor alpha7 subunit is an essential regulator of inflammation. Nature 421: 384-388, 2003.

12. Rittirsch D, Huber-Lang MS, Flierl MA and Ward PA: Immunodesign of experimental sepsis by cecal ligation and puncture. Nat Protoc 4: 31-36, 2009.

13. Livak KJ and Schmittgen TD: Analysis of relative gene expression data using real-time quantitative PCR and the 2(-Delta Delta C(T)) method. Methods 25: 402-408, 2001.

14. Xue P, Huang ZW, Zhang HY, Xia Q, Li YH, Wang ZC, You Z and Guo J: Impact of Chai Qin Cheng Qi decoction on cholinergic anti-inflammatory pathway in rats with severe acute pancreatitis. Sichuan Da Xue Xue Bao Yi Xue Ban 37: 66-68, 2006 (In Chinese).

15. Delong P, Murray JA and Cook CK: Mechanical ventilation in the management of acute respiratory distress syndrome. Semin Dial 19: 517-524, 2006.

15. Davies MG and Hagen PO: Systemic inflammatory response syndrome. Br J Surg 84: 920-935, 1997.

16. Wang H, Vishnubhakat JM, Bloom O, Zhang M, Ombrellino M, Sama A and Tracey KJ: Proinflammatory cytokines (tumor necrosis factor and interleukin 1) stimulate release of high mobility group protein-1 by pituicytes. Surgery 126: 389-392, 1999.

17. Bluth MH, Kandil E, Mueller CM, Shah V, Lin YY, Zhang H, Dresner L, Lempert L, Nowakowski M, Gross R, et al: Sophorolipids block lethal effects of septic shock in rats in a cecal ligation and puncture model of experimental sepsis. Crit Care Med 34: 188-195, 2006. 